\title{
TITLE:
}

\section{$\eta$ 'meson mass from topological charge density correlator in QCD}

\section{$\operatorname{AUTHOR}(\mathrm{S})$ :}

Fukaya, H.; Aoki, S.; Cossu, G.; Hashimoto, S.; Kaneko, T.; Noaki, J.; JLQCD Collaboration

\section{CITATION:}

Fukaya, H....[et al]. n'meson mass from topological charge density correlator in QCD. Physical Review D 2015, 92(11): 111501(R).

\section{ISSUE DATE:}

2015-12-01

URL:

http://hdl.handle.net/2433/250301

RIGHT:

(C) 2015 American Physical Society 
PHYSICAL REVIEW D 92, 111501(R) (2015)

\title{
$\eta^{\prime}$ meson mass from topological charge density correlator in QCD
}

\author{
H. Fukaya, ${ }^{1}$ S. Aoki, ${ }^{2,3}$ G. Cossu, ${ }^{4}$ S. Hashimoto, ${ }^{4,5}$ T. Kaneko, ${ }^{4,5}$ and J. Noaki ${ }^{4}$ \\ (JLQCD Collaboration)
}

\author{
${ }^{1}$ Department of Physics, Osaka University, Toyonaka 560-0043, Japan \\ ${ }^{2}$ Yukawa Institute for Theoretical Physics, Kyoto University, Kyoto 606-8502, Japan \\ ${ }^{3}$ Center for Computational Sciences, University of Tsukuba, Ibaraki 305-8577, Japan \\ ${ }^{4}$ KEK Theory Center, High Energy Accelerator Research Organization (KEK), Tsukuba 305-0801, Japan \\ ${ }^{5}$ School of High Energy Accelerator Science, The Graduate University for Advanced Studies (Sokendai),
}

Tsukuba 305-0801, Japan

(Received 11 September 2015; published 7 December 2015)

\begin{abstract}
The flavor-singlet component of the $\eta^{\prime}$ meson is related to the topological structure of the $\mathrm{SU}(3)$ gauge field through the chiral anomaly. We perform a $2+1$-flavor lattice QCD calculation and demonstrate that the two-point function of a gluonically defined topological charge density after a short Yang-Mills gradient flow contains the propagation of the $\eta^{\prime}$ meson, by showing that its mass in the chiral and continuum limit is consistent with the experimental value. The gluonic correlator does not suffer from the contamination of the pion contribution, and the clean signal is obtained at significantly lower numerical cost compared to the conventional method with the quark bilinear operators.
\end{abstract}

DOI: 10.1103/PhysRevD.92.111501

PACS numbers: $12.38 . \mathrm{Gc}$

Among other hadrons the $\eta^{\prime}$ meson plays a special role in the study of the topological structure of QCD. The $\eta^{\prime}$ meson would be a pseudo-Nambu-Goldstone boson associated with the spontaneous breaking of the axial $U(1)$ symmetry, while it acquires a large mass through the chiral anomaly [1], which relates the divergence of the flavor-singlet axial vector current to the topological charge density in QCD. Witten [2] and Veneziano [3] estimated the $\eta^{\prime}$ meson mass in the large- $N_{c}$ (number of colors) limit and showed that its mass squared is proportional to the topological susceptibility of QCD.

In real QCD with $N_{c}=3$ and light dynamical quarks, the argument of Witten and Veneziano is no longer valid. It is not the $\eta^{\prime}$ meson but the pion that governs the topological susceptibility. In fact, in our previous lattice QCD simulations where we kept the chiral symmetry (nearly) exact [4-6], it was confirmed that the topological susceptibility is proportional to the light sea quark masses as predicted by chiral perturbation theory, $\chi_{t}=\frac{\Sigma}{\sum_{i} 1 / m_{i}}$, where $\Sigma$ denotes the chiral condensate and $m_{i}$ denotes the $i$ th light quark mass. In particular, $\chi_{t}$ vanishes in the limit of massless up and down quarks, reflecting the long-range dynamics of the pion field.

Then, an interesting question arises: what happens to the $\eta^{\prime}$ meson with $N_{c}=3$ ? Since the effect of the anomaly is stronger than in the large- $N_{c}$ limit, the $\eta^{\prime}$ meson mass should be still generated by the topological fluctuation of the gluons. Nevertheless, it must be insensitive to $\chi_{t}$. This implies a nontrivial double-scale structure in the topological excitation of gauge field: it creates the $\eta^{\prime}$ meson at short distances, while making a tight connection to the pion at long distances. The answer to this question could be that the topological property of the $\eta^{\prime}$ meson in QCD is hidden inside the pion clouds.

In this work, by an explicit calculation in 2+1-flavor lattice QCD, we show that the two-point function of the topological charge density at short distances gives a mass consistent with the experimental value of the $\eta^{\prime}$ meson mass. Since we have computed $\chi_{t}$ using the same correlation functions (see Ref. [6] for the details), our data clearly show the double-scale structure of the topological property of gluons: the long-range physics described by the pion and the short-range (or first excited) physics governed by the $\eta^{\prime}$ meson.

Not only is it theoretically interesting, but our work also proposes a practically advantageous method to determine the $\eta^{\prime}$ meson mass. Direct lattice calculation of the $\eta^{\prime}$ meson mass has been challenging because one has to include the disconnected quark-line diagram, which appears from the Wick contraction of the fermion bilinear operators representing the $J^{P C}=0^{-+}$flavor-singlet state, as has been done in recent calculations [7-11]. This is numerically demanding and statistically very noisy.

The advantage of the gluonic calculation adopted in this work over the conventional fermionic one is twofold. First, we can avoid the enormous computational cost of stochastically evaluating the disconnected diagram. The gluonic definition of the topological charge density does not require any inversion of the Dirac operator.

Second, our method avoids the contamination from the pions. In the conventional method where one calculates quark connected and disconnected diagram contributions appearing from the Wick contraction of quark fields, the pion may propagate in each contribution, but it cancels between them. A large statistics is required for a sufficient 
cancellation before extracting the $\eta^{\prime}$ meson propagator. Since the purely gluonic definition of the topological charge density operator

$$
q(x)=\frac{1}{32 \pi^{2}} \epsilon_{\mu \nu \rho \sigma} \operatorname{Tr} F_{\mathrm{cl}}^{\mu \nu} F_{\mathrm{cl}}^{\rho \sigma}(x),
$$

where $F_{\mathrm{cl}}^{\mu \nu}$ denotes the field strength tensor of the gluon field defined through the so-called clover term consisting of four plaquettes, does not directly couple to the pions, its correlator is free from the pion background.

Note here that the sum of (1) over the lattice volume gives the global topological charge up to discretization effects, $Q=\sum_{x} q(x)+\mathcal{O}\left(a^{2}\right)$. However, it is well known that the $\mathcal{O}\left(a^{2}\right)$ contribution is large with currently available lattice spacings. In order to reduce this statistical noise, we modify the link variables using the Yang-Mills (YM) gradient flow [12]. At a flow time $t$, it amounts to smearing the gauge fields in a range of the length $\sqrt{8 t}$. It is shown that the topological charge $Q$ defined through (1) converges to an integer value at a sufficiently large flow time $[13,14]$. This smearing procedure eliminates short-distance fluctuations and also suppresses the noise at longer distances. ${ }^{1}$

For our purpose of extracting the $\eta^{\prime}$ meson mass, the YM gradient flow time has to be short in order not to distort the correlation of the $\eta^{\prime}$ propagation. Assuming a simple Gaussian form of the smearing effect, Bruno et al. [20] estimated the size of distortion of the correlator as

$$
\frac{\Delta\langle q(x) q(y)\rangle}{\langle q(x) q(y)\rangle} \sim e^{-\left(|x-y| / \sqrt{8 t}-m_{\eta^{\prime}} \sqrt{8 t}\right)^{2}} \frac{m_{\eta^{\prime}}(8 t)^{3 / 2}}{2 \sqrt{\pi}|x-y|^{2}} .
$$

In our analysis below, we use the reference flow time around $\sqrt{8 t}=0.2 \mathrm{fm}$ for the fit range $|x-y|>0.6 \mathrm{fm}$, which makes the above correction less than $1 \%$ for $m_{\eta^{\prime}} \simeq 1 \mathrm{GeV}$.

We employ the Symanzik gauge action and the Möbius domain-wall fermion action for gauge ensemble generations [21-23]. We apply three steps of stout smearing of the gauge links before inserting it in the Dirac operator. Our main runs of $2+1$-flavor lattice QCD simulations are carried out on two different lattice volumes $L^{3} \times T=$ $32^{3} \times 64$ and $48^{3} \times 96$, for which we set $\beta=4.17$ and 4.35 , respectively. The inverse lattice spacing $1 / a$ is estimated to be $2.4 \mathrm{GeV}$ (for $\beta=4.17$ ) and $3.6 \mathrm{GeV}$ (for $\beta=4.35$ ), using the input $\sqrt{t_{0}}=0.1465 \mathrm{fm}$ [24] where the reference YM gradient flow time $t_{0}$ is defined by $\left.t^{2}\langle E\rangle\right|_{t=t_{0}}=0.3$ [12], with the energy density $E$ of the gluon field. Our two lattices share a similar physical size $L \sim 2.6 \mathrm{fm}$. For the quark mass, we use two values of the

\footnotetext{
${ }^{1}$ A similar method was applied in a quenched study to extract the "pseudoscalar glueball mass" [15]. There are other viable definitions of the smearing as used in previous works to probe the topological structure of the QCD vacuum [16-19].
}

strange quark mass $m_{s}$ around its physical point and three to four values of the up and down quark mass $m_{u d}$ for each $m_{s}$. The lightest pion mass is around $230 \mathrm{MeV}$ with our smallest value of $a m_{u d}=0.0035$ at $\beta=4.17$. In order to check the systematics due to finite volume sizes and lattice spacings, we also perform simulations on a larger lattice $48^{3} \times 96$ (at $\beta=4.17$ and $m_{\pi} \sim 230 \mathrm{MeV}$ ) and a finer lattice $64^{3} \times 128$ (at $\beta=4.47 \quad[1 / a \sim 4.5 \mathrm{GeV}]$ and $\left.m_{\pi} \sim 285 \mathrm{MeV}\right)$. For each ensemble, 500-1,000 gauge configurations are sampled from 10,000 molecular dynamics (MD) time. The residual mass in the Möbius domainwall fermion formalism is kept smaller than $\sim 0.5 \mathrm{MeV}$ [25] by choosing $L_{s}=12$ at $\beta=4.17$ and $L_{s}=8$ at $\beta=4.35$ (and 4.47).

On each generated configuration, we perform 500-1,000 steps of the YM gradient flow (using the conventional Wilson gauge action) with a step size $a^{2} \Delta t=0.01$. At every 20-30 steps, we store $q(x)$ and measure its correlator using the fast Fourier transform (FFT) technique. As reported in Ref. [6], the flow time history of the gluonic definition of the topological charge $Q$ shows a good convergence to discrete values near integers.

We find that the two-point function $\langle q(x) q(y)\rangle$ at our target distance $|x-y| \sim 0.7$ fm always shows a shorter autocorrelation time than $10 \mathrm{MD}$ time, while its global average, $Q=\sum_{x} q(x)$, has $O(100)$ or higher MD time at $\beta=4.35$. This is a good evidence that the $\eta^{\prime}$ meson physics is decoupled [26] from the global topological charge. In the following analysis, we estimate the statistical error by the jackknife method after binning the data in 140-200 MD time.

Figure 1 shows the topological charge density correlator $C(|x-y|)=-\langle q(x) q(y)\rangle$ at the flow times $a^{2} t=0.2,0.4$ and 1.0. Using the FFT, data points are averaged over all possible combinations of $x$ and $y$ giving the same $r=$ $|x-y|$ to improve the signal. As the flow time increases,

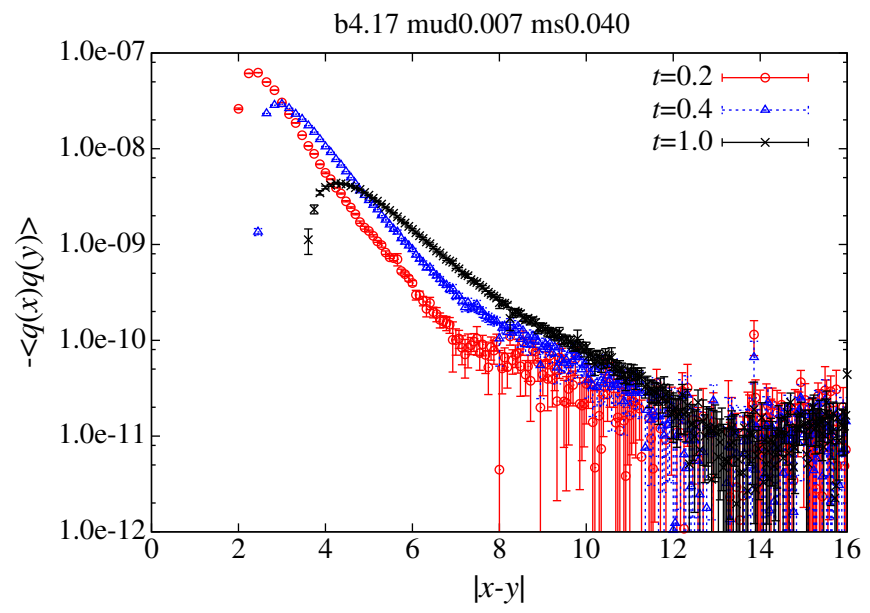

FIG. 1 (color online). The correlator $-\langle q(x) q(y)\rangle$ at the flow times $a^{2} t=0.2$ (circles), 0.4 (triangles) and 1.0 (crosses). Data at $\beta=4.17, a m_{u d}=0.007$ and $a m_{s}=0.040$ are presented. 
$\eta^{\prime}$ MESON MASS FROM TOPOLOGICAL CHARGE ...

the statistical fluctuation of the correlator becomes milder, while the region at small $|x-y|$ is distorted. We therefore need to find a region of $t$ where the correlator has sufficiently small noises to find the signal while it is not distorted by the smearing of the YM gradient flow.

The data for $C(|x-y|)$ show a cleaner signal than the conventional zero-momentum projection, $\sum_{\vec{x}}\langle q(x) q(y)\rangle$, because of the average in all four-dimensional directions. The $\eta^{\prime}$ meson mass is extracted by fitting the data to the function of a single boson propagation:

$$
f\left(r, m_{\eta^{\prime}}\right)=A \frac{K_{1}\left(m_{\eta^{\prime}} r\right)}{r},
$$

where $r=|x-y|, K_{1}$ is the modified Bessel function, and $A$ is an unknown constant, which depends on the flow time $t$.

In order to determine the fitting range, we define a local "effective mass" $m_{\text {eff }}(r)$ by numerically solving $f\left(r+\Delta r, m_{\mathrm{eff}}(r)\right) / f\left(r, m_{\mathrm{eff}}(r)\right)=C(r+\Delta r) / C(r)$. We set the interval to be $\Delta r=1 / 2$, and the data of $C(r)$ in the range $[r, r+\Delta r]$ are averaged. As shown in Fig. 2, a reasonable plateau is found for $m_{\mathrm{eff}}(r)$ around $r \sim 8-12$ $(>0.6 \mathrm{fm})$ at $t=1(\sqrt{8 t} \sim 0.2 \mathrm{fm})$.

Figure 3 shows the $\eta^{\prime}$ meson mass obtained by fitting in the range $[8,12]$ as a function of $\sqrt{8 t}$. The data around $\sqrt{8 t} \sim 0.2 \mathrm{fm}$ are stable. At larger smearing lengths $\sqrt{8 t} \gtrsim 0.3 \mathrm{fm}$, we observe a large distortion of the data. We take the data at $\sqrt{8 t}=0.2-0.25 \mathrm{fm}$ (filled symbols in Fig. 3) for our results.

We plot all the results in Fig. 4 as a function of the square of the pion mass $m_{\pi}$. We find that the dependence on the quark masses, as well as on $V$ and $a$, is mild. We therefore perform a global fit of our data to a linear function $m_{\eta^{\prime}}^{\text {phys }}+$ $C_{a} a^{2}+C_{u d}\left[m_{\pi}^{2}-\left(m_{\pi}^{\text {phys }}\right)^{2}\right]+C_{s}\left[\left(2 m_{K}^{2}-m_{\pi}^{2}\right)-\left\{2\left(m_{K}^{\text {phys }}\right)^{2}-\right.\right.$ $\left.\left.\left(m_{\pi}^{\text {phys }}\right)^{2}\right\}\right]$, where $m_{\eta^{\prime}}^{\text {phys }}, C_{a}, C_{u d}$ and $C_{s}$ are taken as free

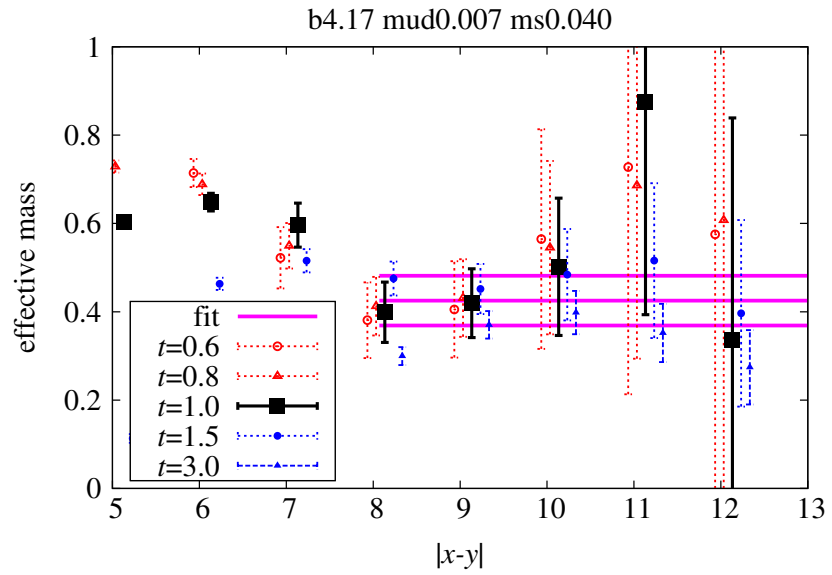

FIG. 2 (color online). The effective mass $m_{\text {eff }}(r)$ for the data with several flow times. Results for the $\beta=4.17 \mathrm{am}_{u d}=0.007$ and $a m_{s}=0.040$ ensemble are shown.
PHYSICAL REVIEW D 92, 111501(R) (2015)

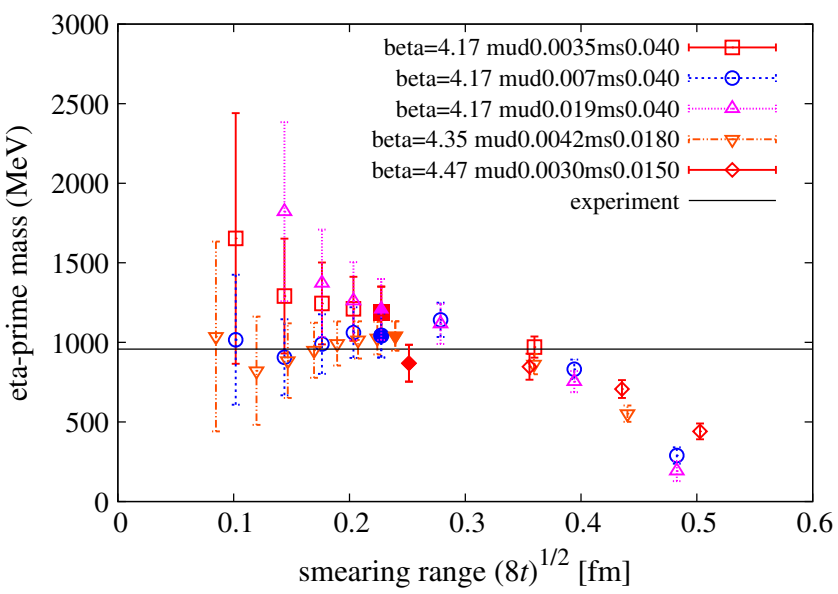

FIG. 3 (color online). The flow-time dependence of the $\eta^{\prime}$ meson mass. The data at various sea quark masses and $\beta$ values are shown, as specified in the legend. The filled symbols represent our data taken for the central values.

parameters and $m_{\pi / K}^{\text {phys }}$ denotes the experimental value of the pion/kaon mass. As shown by the lines (which are shown for higher $m_{s}$ only) in Fig. 4, we find that our linear function fits the lattice data reasonably with $\chi^{2} /($ degrees of freedom $) \sim 1.6$.

Because of possible bias in the topological charge sampling, the $\eta^{\prime}$ correlator may not decay exponentially but become a constant at long distances [27]. In each topological sector $Q$, it is predicted as [28]

$$
\langle q(x) q(y)\rangle^{Q} \sim \frac{1}{V}\left(\frac{Q^{2}}{V}-\chi_{t}\right) \quad \text { at large }|x-y| .
$$

The typical size of the constant term $\chi_{t} / V$ is $100-1000$ times smaller than $|\langle q(x) q(y)\rangle|$ in our fitting range, which

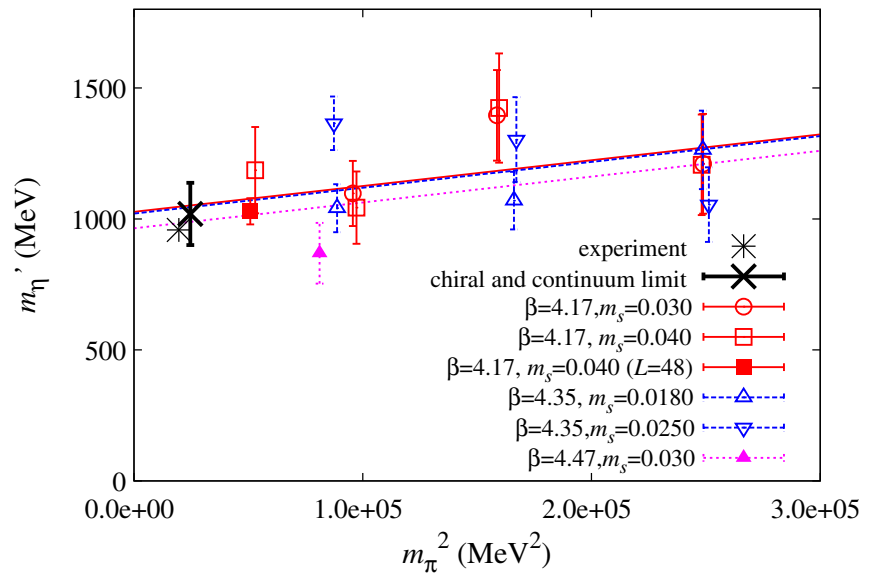

FIG. 4 (color online). The extracted $\eta^{\prime}$ meson mass from each gauge ensemble. The three fit lines (representing the same linear fit function at three values of $a$ ) are shown for higher $m_{s}$ 's at each $\beta$. 
FUKAYA et al.

is consistent with the fact that this observable shows no strong correlation to the global topological charge $Q$.

We also estimate that the finite volume effect on our observable is negligible, since the $\eta^{\prime}$ meson propagator implies $\exp \left(-m_{\eta^{\prime}} L\right) \sim 3 \times 10^{-6}$ even at our lightest $m_{u d}$. Our data on a larger lattice $48^{3} \times 96$, which are statistically consistent with those on the smaller lattice, support this assumption.

Since $m_{u d}$ and $m_{s}$ are nondegenerate, the flavor-singlet channel does not correspond to the mass eigenstate, and a mixing with the flavor nonsinglet channel is expected, which is the $\eta-\eta^{\prime}$ mixing. We estimate the size of the $\eta$ meson contribution to the flavor-singlet channel by modifying the fit function as

$$
\begin{aligned}
\frac{A}{r} K_{1}\left(m_{\eta^{\prime}} r\right) \rightarrow & \frac{A}{r}\left[e^{2 m_{\eta^{\prime}}^{2} t} m_{\eta^{\prime}} K_{1}\left(m_{\eta^{\prime}} r\right)\right. \\
& \left.+e^{2 m_{\eta}^{2} t} m_{\eta} K_{1}\left(m_{\eta} r\right) \tan ^{2} \theta\right],
\end{aligned}
$$

where $\theta$ is the mixing angle and $m_{\eta}$ denotes the $\eta$ meson mass. Note that the factors $e^{2 m_{\eta^{\prime}}^{2} t}$ and $e^{2 m_{\eta}^{2} t}$ come from the effect of the YM gradient flow smearing. As a phenomenological estimate for $m_{\eta}$, we use $\sqrt{m_{\eta_{S}}^{2}\left(m_{u d}+2 m_{s}\right) /\left(3 m_{s}\right)}$ where $m_{\eta_{S}}$ is the (unphysical) mass of the connected pseudoscalar correlator of strange and antistrange quarks [29]. We compare the fit curves with different fixed values of $\theta$ as shown in Fig. 5. As $\theta$ increases the quality of the fit becomes worse, especially at long distances. Thus, our data suggest a small mixing angle $|\theta| \lesssim 10^{\circ}$, and this tendency is seen at all our simulated parameters (the sign of $\theta$ is not relevant for this conclusion). The small mixing angle is consistent with the estimates from the quark model, $\theta=-25^{\circ}--10^{\circ}$ [30]. In our simulations, $\theta$ is likely to be smaller than these phenomenological values since our simulated $m_{u d}$ is closer to $m_{s}$. We confirm that the fit results with the fixed value of $\theta=10^{\circ}$ are consistent with those with $\theta=0$ within the statistical errors, although there exists a tendency that the $m_{\eta^{\prime}}$ meson mass becomes higher for $\theta \neq 0$. We take this $\sim+5 \%$ deviation as the systematic error from the $\eta$ meson mixing.

Finally, we examine the systematics in the chiral and continuum extrapolation. Since both of the $m_{\pi}$ and $a^{2}$ dependences are mild, even if we totally ignore these dependences, the (constant) fit works well, giving a $8 \%$ different value of $m_{\eta^{\prime}}^{\text {phys }}$ from the original linear fit, which is
PHYSICAL REVIEW D 92, 111501(R) (2015)

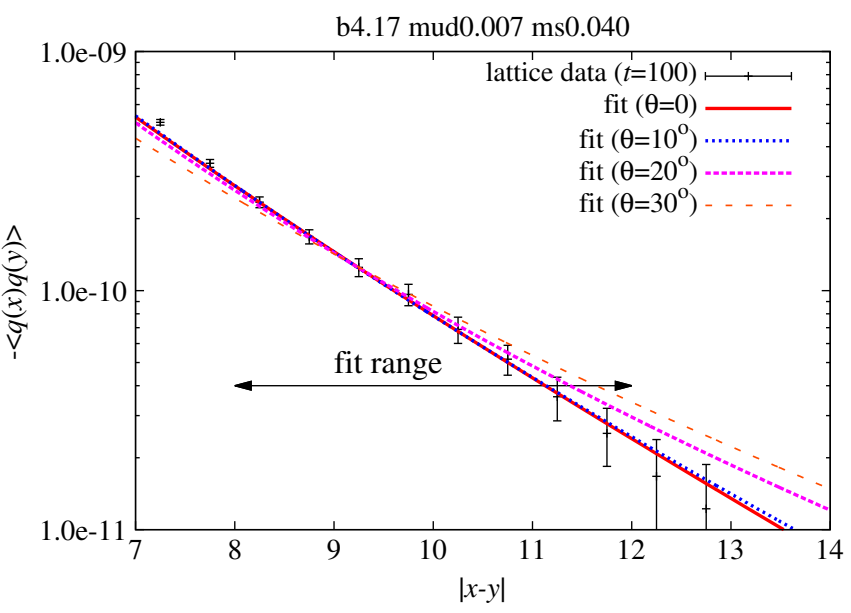

FIG. 5 (color online). Comparison of the fit curves at different inputs $\theta$.

within the statistical error. We take this $\pm 8 \%$ as the possible systematic error in the extrapolations.

Our final result at the physical point is

$$
m_{\eta^{\prime}}=1019(119)\left(\begin{array}{l}
+97 \\
-86
\end{array}\right) \mathrm{MeV},
$$

which is consistent with the experimental value $m_{\eta^{\prime}}=$ 957.78(6) MeV [30]. Here the first error is statistical, and the second is the systematic error from the mixing with the $\eta$ meson and the chiral and continuum extrapolations (added in quadrature). From the same set of correlators we obtain the topological susceptibility $\chi_{t}$, which will be presented elsewhere.

\section{ACKNOWLEDGMENTS}

We thank T. Izubuchi, P. de Forcrand, H. Ohki, and other members of JLQCD Collaboration for fruitful discussions. We also thank the Yukawa Institute for Theoretical Physics (YITP), Kyoto University. Discussions during the YITP workshop YITP-T-14-03 on "Hadrons and Hadron Interactions in QCD" were useful in completing this work. Numerical simulations are performed on IBM System Blue Gene Solution at KEK under the support of its Large Scale Simulation Program (Program No. 14/15-10). This work is supported in part by the Grand-in-Aid of the Japanese Ministry of Education (Grants No. 25287046, No. 25800147, No. 26247043, No. 26400259 and No. 15K05065) and supported in part by MEXT SPIRE and JiCFuS. 


\section{$\eta^{\prime}$ MESON MASS FROM TOPOLOGICAL CHARGE ...}

[1] S. Weinberg, Phys. Rev. D 11, 3583 (1975).

[2] E. Witten, Nucl. Phys. B156, 269 (1979).

[3] G. Veneziano, Phys. Lett. B 95B, 90 (1980).

[4] S. Aoki et al. (JLQCD and TWQCD Collaborations), Phys. Lett. B 665, 294 (2008).

[5] T. H. Hsieh et al. (JLQCD and TWQCD Collaborations), Proc. Sci., LAT2009 (2009) 085.

[6] H. Fukaya et al. (JLQCD Collaboration), Proc. Sci., LATTICE2014 (2014) 323.

[7] T. Kaneko et al. (TWQCD and JLQCD Collaborations), Proc. Sci., LAT2009 (2009) 107.

[8] N. H. Christ, C. Dawson, T. Izubuchi, C. Jung, Q. Liu, R. D. Mawhinney, C. T. Sachrajda, A. Soni, and R. Zhou, Phys. Rev. Lett. 105, 241601 (2010).

[9] E. B. Gregory, A. C. Irving, C. M. Richards, and C. McNeile (UKQCD Collaboration), Phys. Rev. D 86, 014504 (2012).

[10] C. Michael, K. Ottnad, and C. Urbach (ETM Collaboration), Phys. Rev. Lett. 111, 181602 (2013).

[11] K. Ottnad, C. Urbach, and F. Zimmermann (ETM Collaboration), Nucl. Phys. B896, 470 (2015).

[12] M. Lüscher, J. High Energy Phys. 08 (2010) 071; 03 (2014) 092.

[13] M. Lüscher and P. Weisz, J. High Energy Phys. 02 (2011) 051.

[14] C. Bonati and M. D'Elia, Phys. Rev. D 89, 105005 (2014).

[15] A. Chowdhury, A. Harindranath, and J. Maiti, Phys. Rev. D 91, 074507 (2015).

[16] P. de Forcrand, M. G. Perez, and I. O. Stamatescu, Nucl. Phys. B499, 409 (1997).
PHYSICAL REVIEW D 92, 111501(R) (2015)

[17] I. Horváth, A. Alexandru, J. B. Zhang, Y. Chen, S. J. Dong, T. Draper, K. F. Liu, N. Mathur, S. Tamhankar, and H. B. Thacker, Phys. Lett. B 617, 49 (2005).

[18] E.-M. Ilgenfritz, K. Koller, Y. Koma, G. Schierholz, T. Streuer, and V. Weinberg, Phys. Rev. D 76, 034506 (2007).

[19] B. Alles, G. Cossu, M. D’Elia, A. Di Giacomo, and C. Pica, Proc. Sci., LAT2007 (2007) 177.

[20] M. Bruno, S. Schaefer, and R. Sommer (ALPHA Collaboration), J. High Energy Phys. 08 (2014) 150.

[21] T. Kaneko et al. (JLQCD Collaboration), Proc. Sci., LATTICE2013 (2014) 125.

[22] J. Noaki et al. (JLQCD Collaboration), Proc. Sci., LATTICE2013 (2014) 263.

[23] G. Cossu, J. Noaki, S. Hashimoto, T. Kaneko, H. Fukaya, P. A. Boyle, and J. Doi, arXiv:1311.0084; http://suchix.kek .jp/guido_cossu/documents/DoxyGen/html/index.html.

[24] S. Borsanyi et al., J. High Energy Phys. 09 (2012) 010.

[25] S. Hashimoto, S. Aoki, G. Cossu, H. Fukaya, T. Kaneko, J. Noaki, and P. A. Boyle, Proc. Sci., LATTICE2013 (2014) 431.

[26] S. Schaefer, R. Sommer, and F. VirottaS. Schaefer, R. Sommer, and F. Virotta et al. (ALPHA Collaboration), Nucl. Phys. B845, 93 (2011).

[27] G. S. Bali, S. Collins, S. Drr, and I. Kanamori, Phys. Rev. D 91, 014503 (2015).

[28] S. Aoki, H. Fukaya, S. Hashimoto, and T. Onogi, Phys. Rev. D 76, 054508 (2007).

[29] JLQCD Collaboration (unpublished).

[30] J. Beringer et al. (Particle Data Group Collaboration), Phys. Rev. D 86, 010001 (2012); K. A. Olive et al. (Particle Data Group), Chin. Phys. C 38, 090001 (2014). 